\title{
Timing Properties of Magnetars
}

\author{
Feryal Özel ${ }^{1}$ \\ Harvard-Smithsonian Center for Astrophysics and Physics Department, Harvard University, \\ 60 Garden St., Cambridge, MA 02138; fozel@cfa.harvard.edu
}

\begin{abstract}
We study the pulse morphologies and pulse amplitudes of thermally emitting neutron stars with ultrastrong magnetic fields. The beaming of the radiation emerging from a magnetar was recently shown to be predominantly non-radial, with a small pencil and a broad fan component. We show that the combination of this radiation pattern with the effects of strong lensing in the gravitational field of the neutron star yields pulse profiles that show a qualitatively different behavior compared to that of the radially-peaked beaming patterns explored previously. Specifically, we find that: (i) the pulse profiles of magnetars with a single hot emission region on their surface exhibit $1-2$ peaks, whereas those with an antipodal emission geometry have $1-4$ peaks, depending on the neutron star compactness, the observer's viewing angle, and the size of the hot regions; (ii) the energy dependence of the beaming pattern may give rise to weakly or strongly energy-dependent pulse profiles and may introduce phase lags between different energy bands; (iii) the non-radial beaming pattern can give rise to high pulsed fractions even for very relativistic neutron stars; (iv) the pulsed fraction may not vary monotonically with neutron star compactness; $(v)$ the pulsed fraction does not decrease monotonically with the size of the emitting region; (vi) the pulsed fraction from a neutron star with a single hot pole has, in general, a very weak energy dependence, in contrast to the case of an antipodal geometry. Comparison of these results to the observed properties of anomalous X-ray pulsars strongly suggests that they are neutron stars with a single hot region of ultrastrong magnetic field.
\end{abstract}

Subject headings: radiation mechanisms:thermal — stars:magnetic fields — stars:neutron - X-rays:stars

\section{Introduction}

Anomalous X-ray Pulsars (AXPs) and Soft Gamma-ray Repeaters (SGRs) are two classes of intriguing objects that have challenged the standard paradigm of young neutron stars. Particularly

\footnotetext{
${ }^{1}$ Present address: Institute for Advanced Study, Einstein Dr., Princeton, NJ 08540
} 
in the case of AXPs, two types of models have been proposed to explain their spectral and timing properties: conventional accretion models modified to account for the absence of an observable donor star (van Paradijs, Taam, \& van den Heuvel 1995; Chatterjee, Hernquist, \& Narayan 2000; Alpar 2001) and magnetar models with several different mechanisms for powering the X-ray emission (Thompson \& Duncan 1996; Heyl \& Hernquist 1998). In all these magnetar models, a significant fraction of the X-ray emission is thought to originate at the neutron star surface.

Recently, there has been significant progress in the calculation of the surface emission properties of ultramagnetized neutron stars and their application to AXPs (Özel 2001; Özel, Psaltis, \& Kaspi 2001; see also Ho \& Lai 2001; Zane et al. 2001). Özel (2001) showed that the angle dependence of surface radiation in such strong fields has both a pencil and a fan component, with the latter dominating in the X-ray range. Such a beaming pattern can give rise to a variety of pulse profiles and timing properties that have not been explored to date. Furthermore, the general relativistic bending of photon trajectories in the strong gravitational field of the neutron star significantly affects the observed properties of emission originating at the stellar surface. This is in contrast to rotation-powered pulsars whose radio emission originates at the light cylinder far above the stellar surface, where the self lensing by the neutron star is weak. Therefore, in order to compare in detail the models with the current and future observations of AXPs, it is now necessary to carry out a complete study of the observable properties of magnetars taking all the above effects into account.

The advent of X-ray telescopes with good timing and energy resolution as well as broad spectral coverage has produced high-quality data on AXPs in the recent years, making such a study timely. Indeed, observations with $A S C A$ and BeppoSAX have yielded good broad-band spectra of AXPs as well as a measure of the flux variations during a pulse cycle in multiple energy bands (e.g., White et al. 1996; Oosterbroek et al. 1998). Moreover, the superb timing resolution of the Rossi $X$-ray Timing Explorer ( $R X T E$ ) made possible the phase connection of pulse cycles over several years and hence produced detailed energy-dependent pulse profiles (Gavriil \& Kaspi 2002). Finally, the grating spectrographs onboard the Chandra and XMM-Newton observatories extend the energy range of current observations towards the soft X-rays in addition to allowing for phase-resolved spectroscopy and searches for spectral lines (e.g., Patel et al. 2001; Juett et al. 2002; Tiengo et al. 2002). It has been argued that the combination of the pulse profiles, the amplitude of pulsations, and the pulse-phase resolved spectral features observed with these instruments provide the most stringent constraints on the models of AXPs (Özel et al. 2001).

In this paper we explore the pulse profiles expected from a strongly magnetized neutron star emitting thermally from its hot surface. In detail, we focus on the dependence of the pulse profiles on the emission geometry, the orientation of the observer, the photon energy, and the compactness of the neutron star. We also discuss the implications of our results for magnetar models of AXPs. 


\section{The Model}

We consider the emission from a hot ultramagnetized neutron star. In order to determine the properties of its surface emission, we carry out radiative transfer calculations in strong fields and construct model atmospheres in radiative equilibrium. We assume that the stellar atmosphere is a completely ionized electron-proton plasma in plane-parallel geometry, owing to its negligible thickness compared to the stellar radius. We take the magnetic field to be orthogonal to the surface, motivated by the fact that the emission most probably originates at the magnetic poles. We consider fully angle- and energy-dependent emission, absorption, and scattering processes in the two photon polarization modes of a magnetized plasma and include the effects of vacuum as well as of the plasma on photon propagation. We neglect the effects of the neutron star magnetosphere which may affect the photon interactions above the stellar atmosphere. A detailed description of the model as well as the surface emission properties are given in Özel (2001).

There are two modifications in the present calculation regarding the treatment of vacuum polarization, which have a weak effect on the results. First, for magnetic field strengths above the critical value $B_{\text {cr }}=4.414 \times 10^{13} \mathrm{G}$, we modify the interaction cross sections following Tsai \& Erber (1975). Second, we employ an adaptive mesh technique to resolve the sharp features associated with the vacuum polarization resonance instead of the saturation technique presented in Özel (2001). The details of these improvements as well as the effects of the proton cyclotron resonance on the emerging spectra are discussed in Özel (2002).

We consider two emission geometries: (i) a single hot pole that corresponds to, e.g., an isolated region of enhanced magnetic activity, and (ii) two antipodal hot regions corresponding to, e.g., two magnetic poles. In both cases, the temperature is taken to be uniform across the hot region and is specified by an effective temperature $T_{\mathrm{e}}$, while the rest of the neutron star is assumed not to radiate. We allow for variations of the angular size of the emitting region $\rho$ as well as of the orientation angles $\alpha$, which specifies the location of the magnetic poles with respect to the rotation axis on the neutron star surface, and $\beta$, which determines the position of the observer with respect to the same axis. For most of the calculations presented here, we fix the magnetic field strength to $B=10^{15} \mathrm{G}$. We also use an effective temperature $T_{\mathrm{e}}=0.5 \mathrm{keV}$, which yields spectra with color temperatures at infinity of $\approx 0.5-0.6 \mathrm{keV}$ at this field strength, appropriate for the spectral characteristics of AXPs (see Özel et al. 2001).

In calculating the observables at infinity, we take into account the gravitational lensing of the surface emission by the neutron star. We use the photon trajectories given by the Schwarzschild metric, which provides a good approximation for slowly rotating neutron stars such as AXPs, and follow the method described by Pechenick, Ftaclas, \& Cohen (1983) to compute light curves. We vary the neutron star relativity parameter $p=R c^{2} / 2 G M$, where $R$ and $M$ denote the radius and

the mass of the neutron star, respectively. We consider the range $2 \leq p \leq 4$, which is allowed by current equations of state for a wide range of neutron star masses (see, e.g., Cardall, Prakash, \& Lattimer 2001). Note that the magnetic pressure becomes important in hydrostatic balance for 


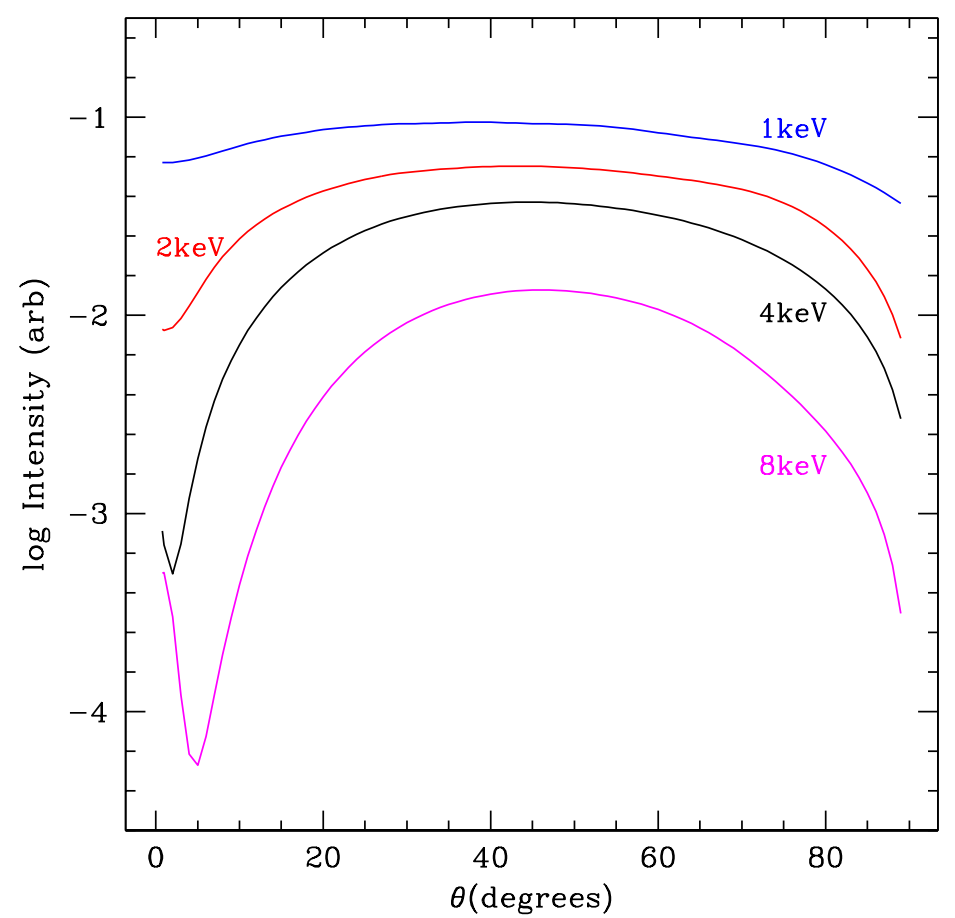

Fig. 1. - The angle dependence (beaming) of radiation emerging from the surface of a neutron star with $B=10^{15} \mathrm{G}$, at photon energies of $1,2,4$ and $8 \mathrm{keV}$.

very large $\left(B \sim 10^{17} \mathrm{G}\right)$ field strengths and may lead to larger neutron star radii in this regime. However, because the magnetic field does not significantly affect the equation of state at the lower values considered here, we take the often employed value of $p=2.5$ corresponding to a $1.4 M_{\odot}$ and $10 \mathrm{~km}$ neutron star as the fiducial value when varying the other model parameters.

\subsection{The Beaming of Surface Emission}

The pulse profiles are determined primarily by the angle dependence (beaming) of the radiation emerging from the neutron star surface and the general relativistic bending of photon trajectories in strong gravitational fields. In the ultrastrong magnetic fields that we consider here, the beaming is qualitatively different than that of weakly-magnetic, non-magnetic, or accreting neutron stars and is predominantly non-radial. A detailed discussion of the beaming of surface radiation from a magnetar is given in Özel (2001). Here, we only summarize the results to facilitate the discussion of the pulse profiles.

Figure 1 shows the beaming of radiation emerging from the surface of a neutron star with $B=10^{15} \mathrm{G}$ and $T_{\mathrm{e}}=0.5 \mathrm{keV}$, at photon energies $E=1,2,4$, and $8 \mathrm{keV}$. The abscissa $\theta$ denotes the angle between the direction of the magnetic field and the direction of propagation. In all cases, the 


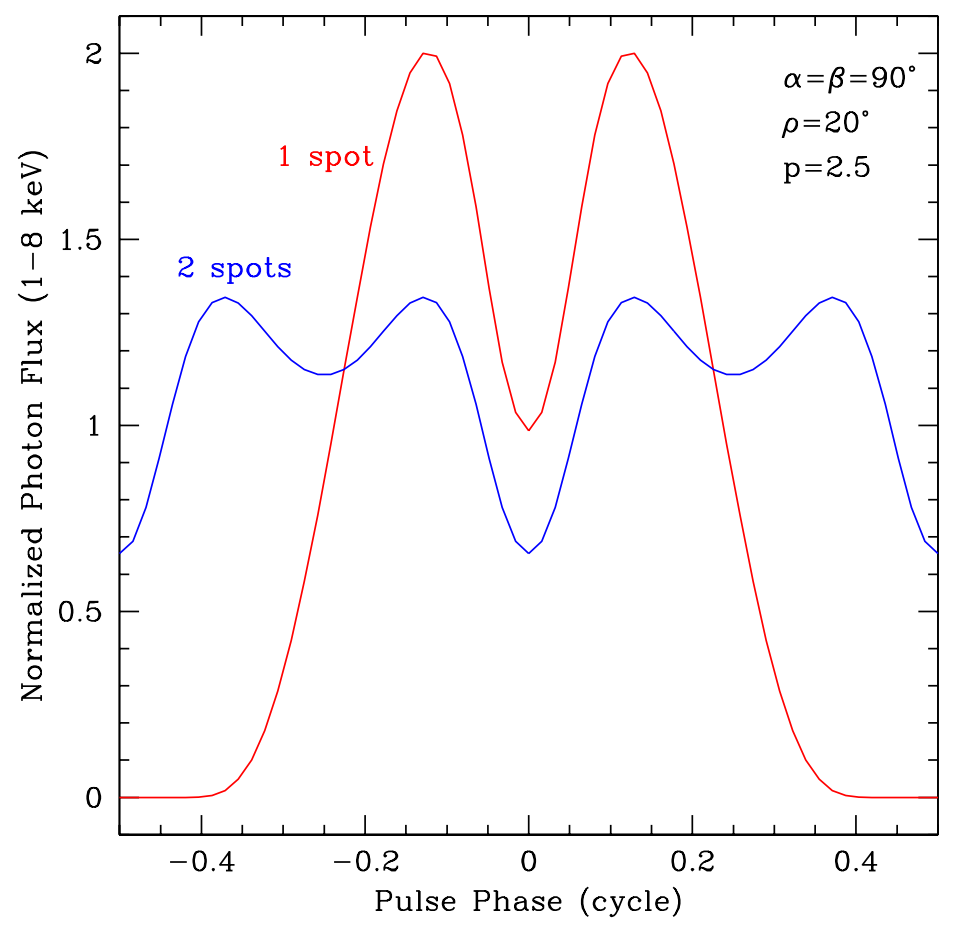

Fig. 2.- Sample $1-8 \mathrm{keV}$ pulse profiles for a neutron star with a single or two antipodal hot regions. The former case shows two and the latter four peaks per pulse cycle when the observer traverses the emission cones at the center.

radiation pattern can be described by two components: a radial beam confined to $\theta \lesssim$ few degrees and a dominant wide "fan" beam which has a peak at $\theta_{\mathrm{p}} \approx 40^{\circ}-60^{\circ}$. The radial beam carries little flux and is observationally insignificant because of its confinement to very small angles. Therefore, the pulse profiles, and in particular the number and location of the peaks, carry signatures of the fan beams. Note that when viewed along the magnetic axis on the neutron star surface, this beaming pattern represents a hollow cone of radiation whose opening angle and thickness vary with photon energy.

The beaming of radiation shown in Figure 1 varies weakly with magnetic field in the range $10^{14}-10^{15} \mathrm{G}$. Therefore, the pulse profiles do not change significantly in this range of field strengths.

\section{Bolometric Pulse Profiles}

In this section, we present energy-integrated pulse profiles of magnetars emitting thermally from their surface. We choose a $1-8 \mathrm{keV}$ photon energy range, which is accessible with high sensitivity with the current detectors and is thus relevant for comparison with the present data on AXPs. For simplicity, we refer to quantities computed in this energy range as bolometric. We 
focus on the number and location of the peaks in the pulse profile, as well as on the peak-to-peak changes of the flux through a pulse cycle. We will consider the photon-energy dependence of the profiles and further quantify the modulation (through the use of the pulsed fraction) in $\S 4$ and $\S 5$, respectively.

Figures 2-4 show bolometric pulse profiles from a $B=10^{15} \mathrm{G}$ neutron star with $T_{\mathrm{e}}=0.5 \mathrm{keV}$. The ordinate shows the photon flux normalized to the average flux through a pulse cycle, while in the abscissa $\phi=0$ corresponds to the phase when the observer is aligned with the center of one pole. The pulse profiles shown in these figures are determined primarily by the beaming of radiation on the neutron star surface as well as by the strong gravitational lensing by the neutron star. In three dimensions, the non-radial beaming pattern (Fig. 1) corresponds to a bright hollow cone of radiation with angular size $\sim \theta_{p}$ and thickness $\approx 30^{\circ}$ originating from every surface element on the neutron star. Thus, in the absence of gravitational lensing and for an observer traversing the diameter of a small emitting region, two peaks appear per hot pole, at a phase that corresponds to the location of the peaks in the beaming pattern. Thus, the phase difference between the peaks originating from one pole is approximately $2 \theta_{p}$. Figure 2 shows the pulse profiles for this simplest case for the two emission geometries discussed in $\S 2$ : a single pole gives rise to 2 peaks while an antipodal emission geometry produces 4 peaks.

As the above discussion suggests, in more realistic cases, the properties of the pulse profiles are determined not only by the number of hot regions on the stellar surface but also by (i) general relativistic effects, (ii) the orientation angles that describe the positions of the emitting regions and of the observer with respect to the rotation axis and (iii) the sizes of the emitting regions. We now explore each of these effects in detail, investigating the resulting number and separation of the peaks in the pulse profiles and the peak-to-peak changes of the flux during a pulse phase.

\subsection{The Effect of the Gravitational Lensing by the Neutron Star}

General relativistic effects spread out and smear the beaming patterns, effectively changing the observed opening angles and thicknesses of the hollow radiation cones. In general, self-lensing by the neutron star makes more of the stellar surface visible to the observer at any pulse phase and leads to a suppression of the modulation of the observed lightcurves. In some cases, however, non-radial

beams can be focused away from the surface normal by the gravitational lensing in a way that the flux at particular pulse phases can be enhanced. This happens when cones of radiation originating from different parts of one hot region or from two antipodal hot regions are deflected towards each other and smeared so strongly by the stellar gravity that they overlap. This effect can also change the number of peaks in the pulse profile. Thus, the general relativistic effects in conjunction with a predominantly non-radial radiation pattern (fan beaming) give rise to qualitatively different results, affecting the number and separation of the peaks in the pulse profile as well as the overall flux modulation. 

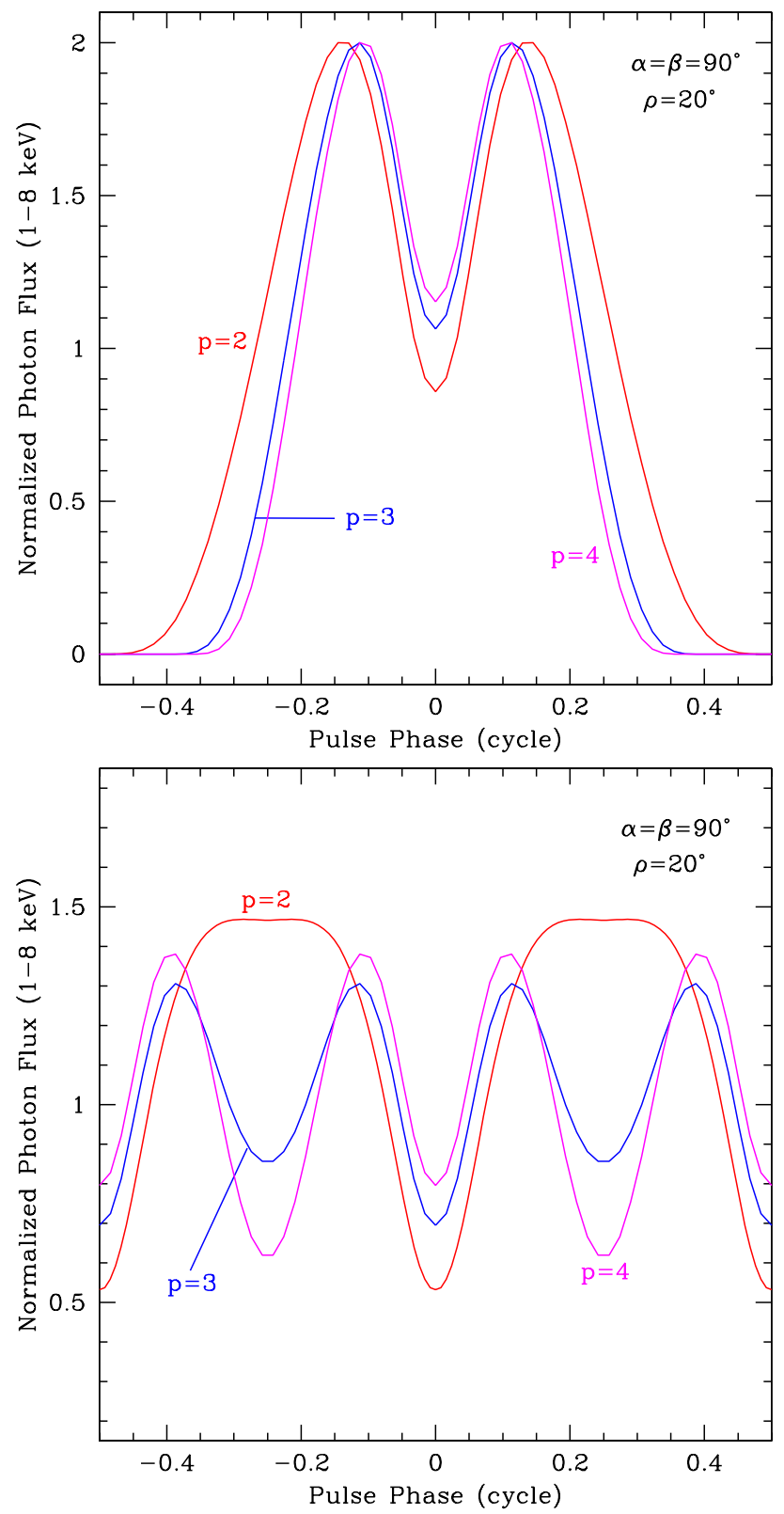

Fig. 3. - The $1-8 \mathrm{keV}$ pulse profiles for varying neutron star compactness $R / 2 M=2,3$ and 4 . The neutron star has a magnetic field of $10^{15} \mathrm{G}$ and an effective temperature $T_{\text {eff }}=0.5 \mathrm{keV}$. The top panel shows the result for a single hot pole while the bottom panel corresponds to an antipodal emission geometry, with an angular size of the hot regions $\rho=15^{\circ}$ in both cases. The two orientation angles $\alpha$ and $\beta$ are taken to be $90^{\circ}$ (orthogonal rotator).

Figure 3 shows the effect of varying the relativity parameter $p$ on the pulse profiles. The $p=4$ case represents a large neutron star where the curvature of photon paths is small, and the result is 

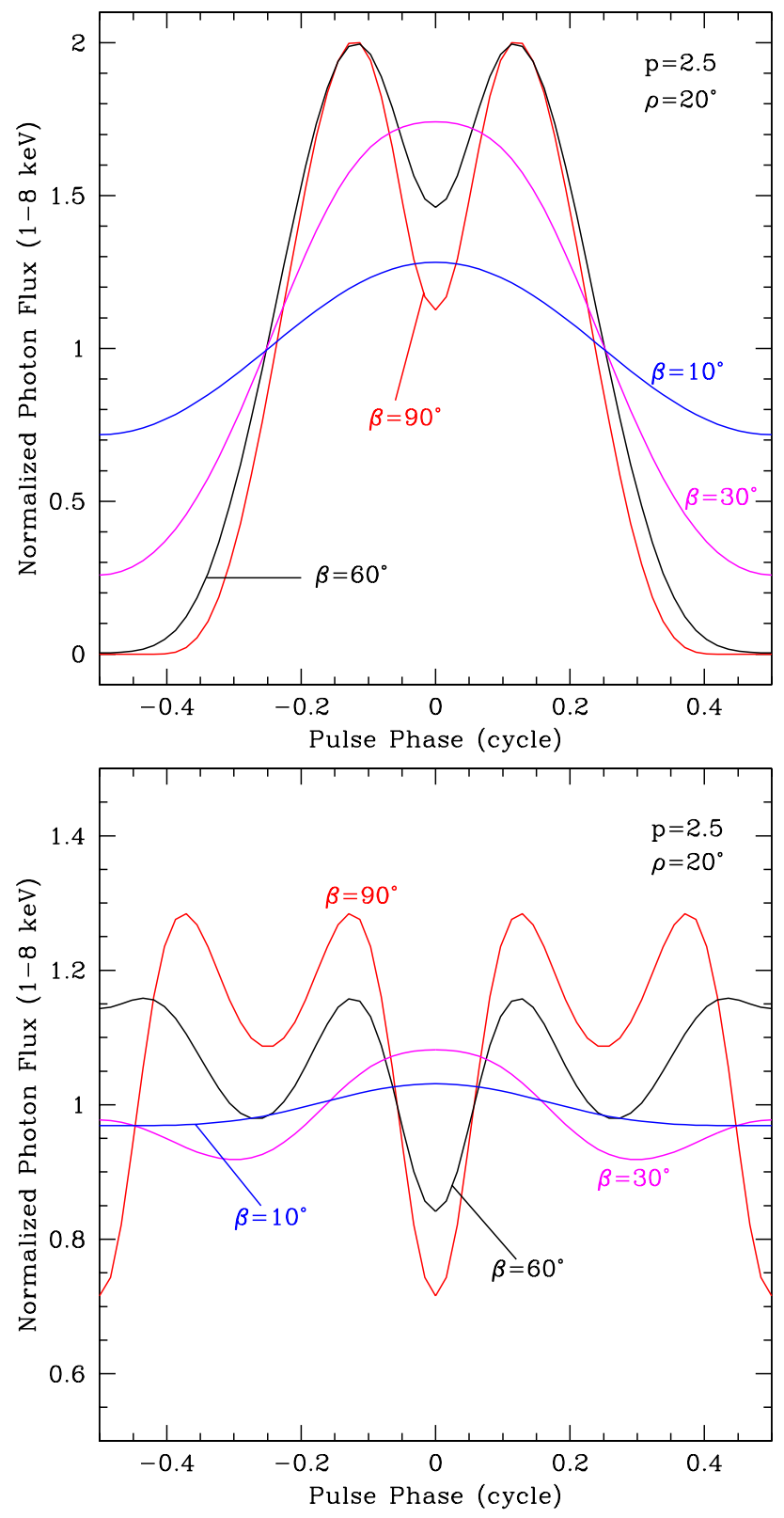

Fig. 4. - The $1-8 \mathrm{keV}$ pulse profiles for different values of the observer angle $\beta$. Here, $\alpha=80^{\circ}$ while the field strength and effective temperature are as in Figure 3. The top panel shows $1-2$ peaks arising from a single pole and the bottom panel displays the $1-4$ peaks that can be obtained in the antipodal emission geometry.

nearly Newtonian with 4 peaks located roughly at phases $\phi= \pm \theta_{\mathrm{p}}$ and $\pi \pm \theta_{\mathrm{p}}$. As $p$ decreases, i.e., as the neutron star becomes more relativistic, gravitational lensing becomes stronger. The beams detected by an observer at infinity are correspondingly defocused and appear with larger angular 
sizes. Therefore, the observed peaks in the pulse profile get broader and move to phases $\phi>\theta_{p}$. Another consequence is that more flux reaches the observer during the interpulses, as in the case of $p=3$ shown in Figure 3.

As the neutron star becomes even more relativistic, pulse profiles in the antipodal emission case go through a significant morphological change because the cones of emission from the antipodal hot regions start to overlap and produce an enhanced signal at $\phi= \pm \pi / 2$. When the beams combine in this way, the number of peaks in the pulse profile is reduced and the overall modulation is increased (Fig. 3). Note that for small values of $p$, the same effect can be seen in the emission from a single pole, when its area is large enough for the beams from two sides of the hot region to combine (also see $\S 3.3$ and Fig. 6 below). In that case, the number of peaks in the pulse phase is reduced

from two to one. To summarize, the harmonic structure and modulation of the pulse profiles are directly determined by the neutron star compactness, with a sharp transition in the properties of the profiles at small relativity parameters $p$. For most values of $\alpha, \beta$ and $\rho$, this transition in the pulse profiles occurs at $p \simeq 2.5$.

\subsection{The Effect of Geometry: Orientation Angles}

The orientation angles $\alpha$ and $\beta$ determine two aspects of the pulse profiles. First, because they indicate the position of the poles and of the observer with respect to the rotation axis, they control the overall modulation of the lightcurves, ranging from no modulation at $\alpha=0$ or $\beta=0$ to maximum modulation at $\alpha=\beta=90^{\circ}$. Second, their relative values determine the chord that the observer's path cuts through the cone of emission, and thus the observed number and separation of peaks in the pulse profile.

Figure 4 shows the pulse profiles for different values of the observer's angle $\beta$. Here, we fix the position angle $\alpha$ to $80^{\circ}$ and vary $\beta$ between $10^{\circ}$ and $90^{\circ}$ for an emitting region of size $\rho=20^{\circ}$ and a relativity parameter $p=2.5$. The striking result is that the number of peaks can vary between $1-2$ for the single pole and between $1-4$ for the antipodal emission geometry, determined by the number of times the observer intersects the cones of emission. However, unlike the case of neutron star compactness, which also affects the observed number of peaks, the reduction from four peaks to one due to different observer's angles is usually accompanied by a suppression in the pulsed fraction.

In Figure 5, we identify the regions in the $(\cos \alpha, \cos \beta)$ parameter space that produce different number of peaks in the observed pulse profiles, the top panel corresponding to a single pole and the bottom panel to the antipodal emission geometry. For a random distribution of orientation angles, the areas covered by each region is directly proportional to the probability of observing a system with a given number of peaks in its pulse profile. 

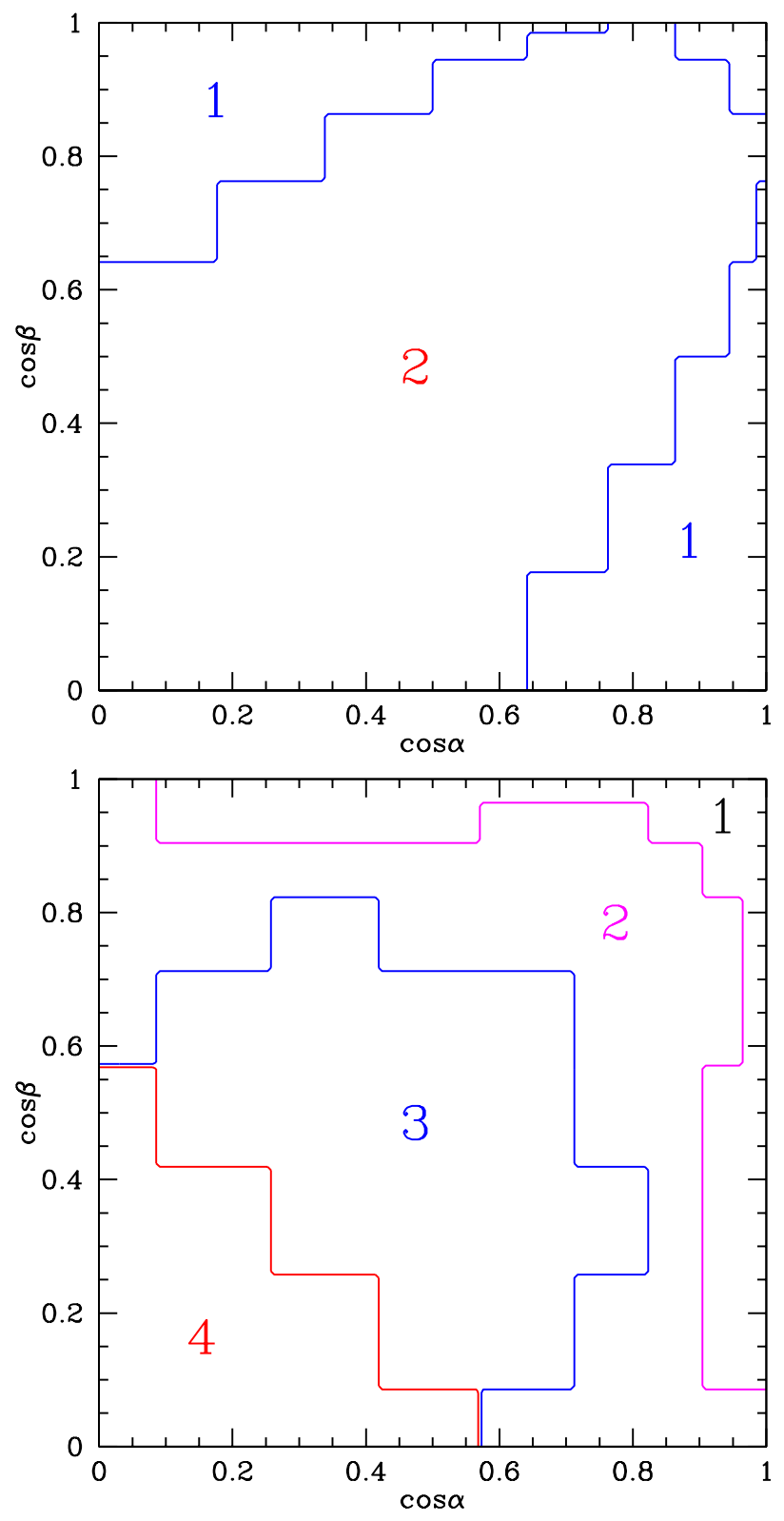

Fig. 5.- The contours show the number of peaks in the pulse profile as a function of the cosine of the orientation angles $\alpha$ and $\beta$ for a single pole (top panel) and the antipodal geometry (bottom panel). All other model parameters are as in Figure 4.

\subsection{The Effect of the Size of the Hot Regions}

The last parameter that affects significantly both the number of peaks and the modulation of the pulse profiles is the area covered by the hot regions on the stellar surface. Figure 6 shows the dependence of the pulse profiles on the size of the emitting region for both the single and antipodal 
emission geometries. As discussed earlier, for large emitting regions, the observer receives the most flux at a phase when the line of sight coincides with the center of the pole because the non-radial emission from the outer edges of the pole is directed towards the center. This enhancement reduces the number of peaks to one in the case of the single pole while it shifts the phase of the peaks by $\approx \pi / 4$ in the antipodal emission case.
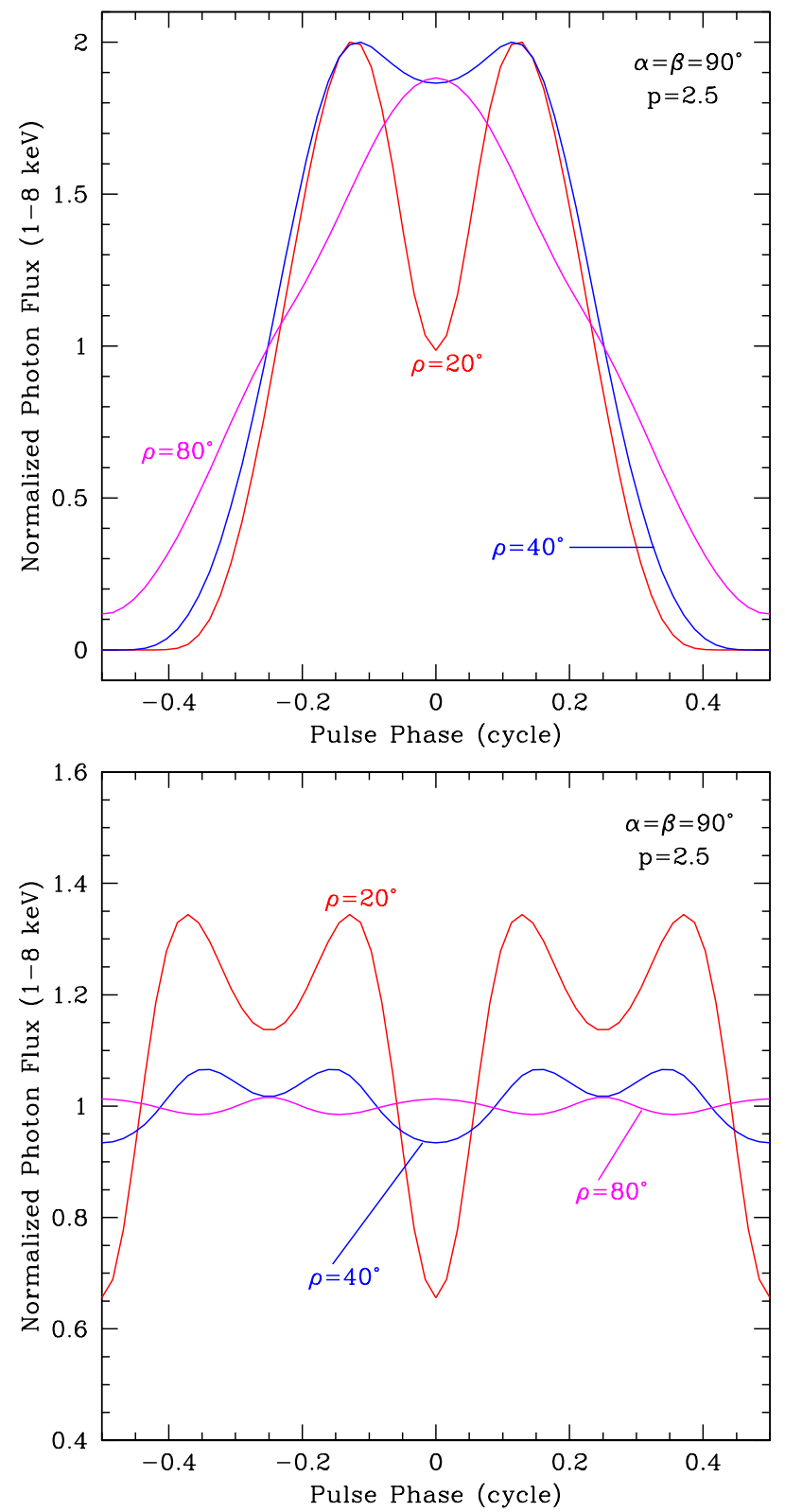

Fig. 6. - The dependence of the pulse profiles on the size of the emitting region for (top panel) a single pole and (bottom panel) two antipodal hot regions on the neutron star. All other model parameters are the same as before. 


\section{Photon-Energy Dependence of the Pulse Profiles and Phase Lags}

In this section, we investigate the dependence of pulse profiles on photon energy in an energy range of interest for observations of AXPs. The pulse profiles of strongly magnetized neutron stars are expected in general to have a dependence on photon energy because of the different beaming of the emerging radiation at different photon energies. However, the properties of the pulse profiles

in different energy bands are also affected strongly by the number and sizes of the emitting regions as well as by the orientation angles $\alpha$ and $\beta$, as we show below. Following Gavriil \& Kaspi (2002), we choose the energy ranges $1-2,2-4$, and $6-8 \mathrm{keV}$ for this study.

Because the photon-energy dependence of the pulse profiles changes with all of the parameters that specify the geometry of the surface temperature distribution and the position of the observer, it is not feasible to illustrate the pulse profiles for the entire space spanned by these parameters. Instead, we show in Figures 7 - 10 some representative cases. In particular, the examples include cases where the dependence of the pulse profiles and/or pulse amplitudes on photon energy is either very weak or rather strong. We discuss the range of parameters for which each behavior can be observed.

Figure 7 shows the case of an orthogonal rotator with one and two $20^{\circ}$ hot poles (top and bottom panels respectively) on a neutron star with compactness $p=2.5$. For this set of parameters, the energy dependence in the single pole case is quite weak and is characterized by a small phase shift in the location of the peak of the pulse profiles between different energies. In addition, there is a more prominent interpulse (at $\phi=0$ in Fig. 7) at low photon energies. The pulse amplitudes are nearly equal in the three bands. This type of weak dependence on photon energy is very common in the single-pole geometry and is obtained for most of the model parameters.

The slight phase shift and broader pulses are a direct consequence of the different beaming of radiation at different photon energies. As shown earlier in Figure 1, the peak of the fan beam appears at smaller angles $\theta_{\mathrm{p}}$ for smaller photon energies, giving rise to narrower emission cones. Furthermore, at these lower energies, the beams are flatter and the minimum separating the fan and pencil beams becomes less prominent. Thus, for the orthogonal rotator geometry, where an observer intersects the emission cones at the diameter, the lower energy peaks appear more closely spaced than the higher energy peaks and the flux between them is higher.

In the antipodal emission geometry, the pulse shapes in the different energy bands are also similar and the small phase shift in the location of the peaks is present. On the other hand, there is a marked difference in the pulse amplitudes in the different energy bands. Note that Figure 7 shows one of the weakest energy dependences in the pulse morphology that can be obtained for any choice of parameters in this emission geometry.

Figure 8 shows energy-dependent pulse profiles for different values of the observer's angle $\beta$ and the angular size of the emitting area $\rho$. In these examples, the energy dependence is stronger, both in the pulse shapes and in the pulse amplitudes. In the case of one pole, the cross-correlation of the $1-2$ and $6-8 \mathrm{keV}$ pulse profiles reveals an apparent phase lag of half a cycle caused primarily by 

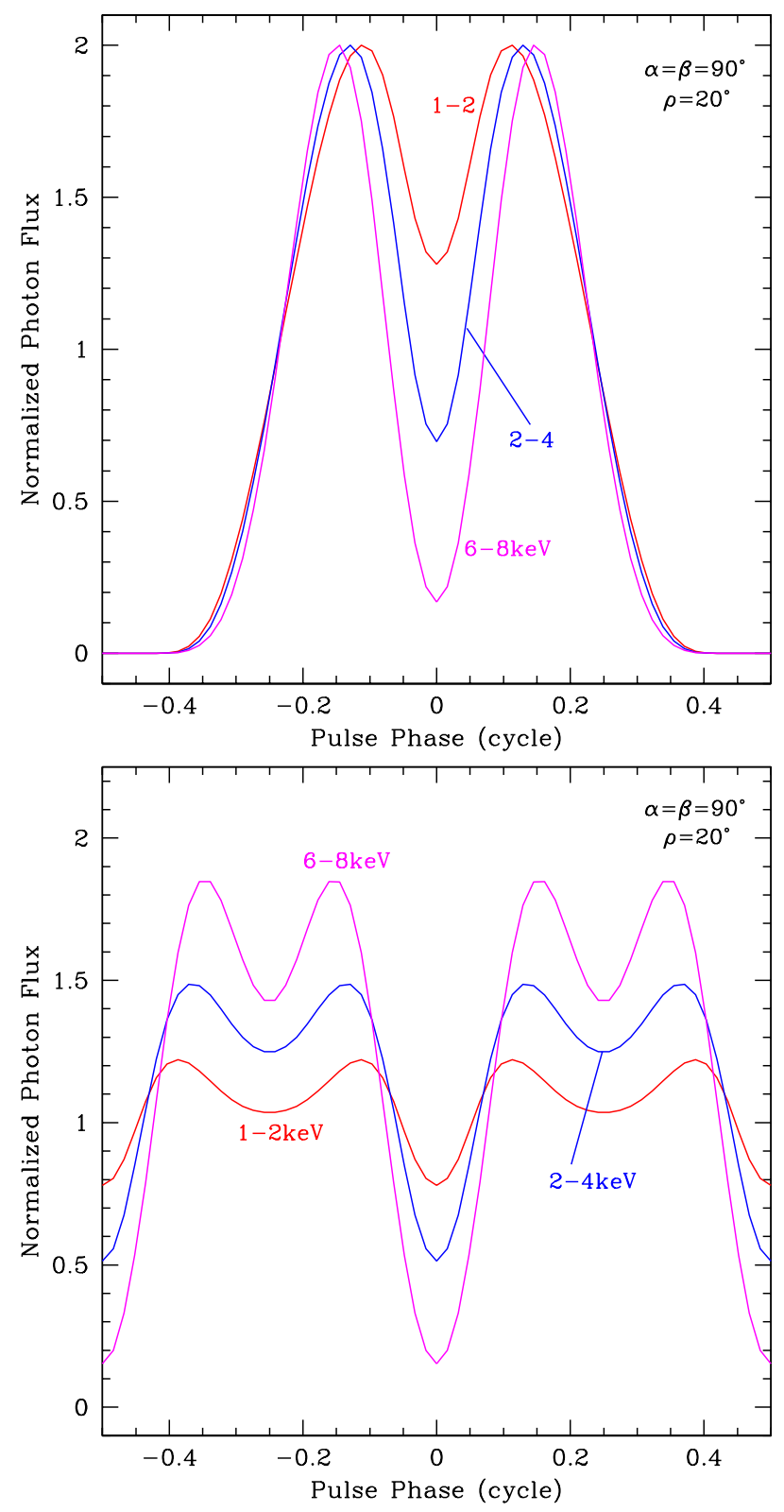

Fig. 7.- Pulse profiles that have rather similar morphologies in different energy bands between 1-8 keV. The top panel shows the results for a single pole while the bottom panel shows the results for an antipodal emission geometry.

the difference in the phase of the minima in the two profiles. In the antipodal geometry, the number of peaks increases by one in the higher photon-energy band. In both cases, the pulse amplitude is highest in the $6-8 \mathrm{keV}$ band. Such simultaneous changes in both the pulse morphology and in the pulse amplitude are rare in the case of a single pole but are obtained for most of the parameter space for the antipodal geometry. 

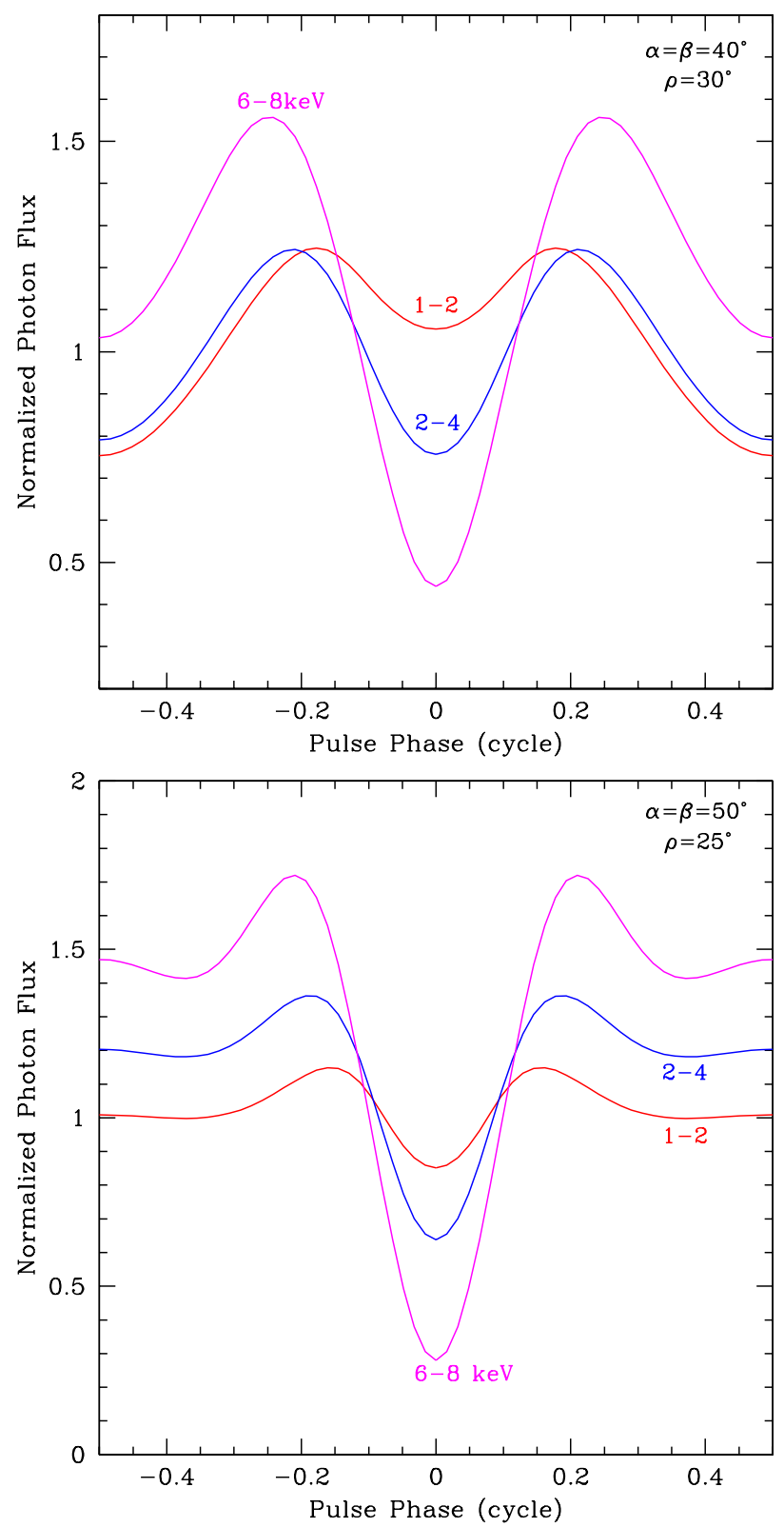

Fig. 8.- A strong photon-energy dependence of the pulse profiles between 1-8 keV. The top panel corresponds to a single and the bottom panel to two antipodal hot regions on the neutron star surface.

The bottom panel of Figure 8 along with that of Figure 7 spans the entire range of energydependent pulse profiles that can arise in the antipodal emission geometry. In the case of a single hot pole, on the other hand, the energy dependence of the pulse profiles shows more diversity, as the following examples illustrate. 


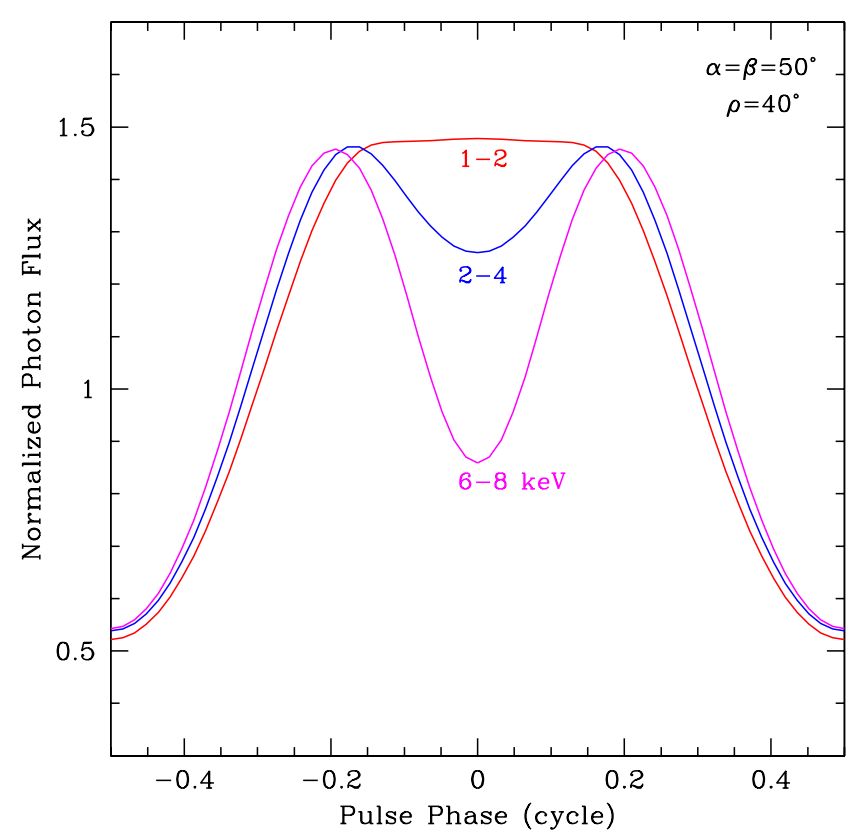

Fig. 9.- An example of a pulse profile at different photon energies from a magnetar with a single hot pole, where the pulse shapes are energy-dependent while the pulse amplitudes remain constant.

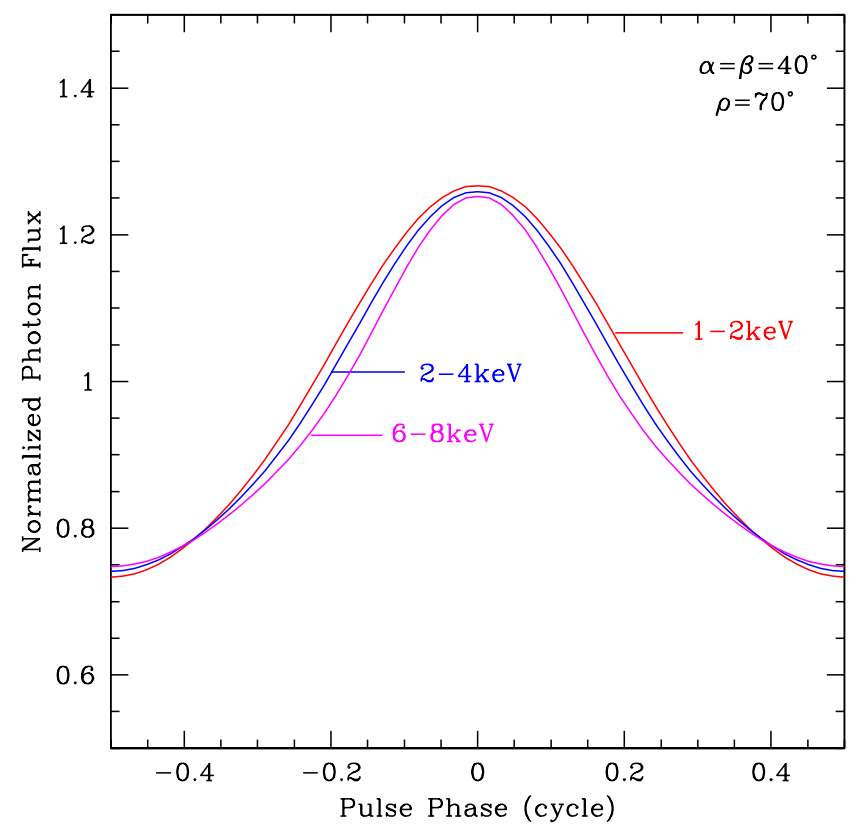

Fig. 10. - An example of a singly-peaked pulse profile at different photon energies from a magnetar with a single hot pole, where the pulse shapes and amplitudes show no energy dependence. 
Figure 9 shows a configuration where the pulse morphology, and in particular the number of peaks, changes with photon energy, while the pulse amplitude remains constant. Finally, Figure 10 shows a pulse profile with a single peak and no energy dependence. This is obtained when the observer grazes the emission cones at all photon energies. Such a case is relevant to the observations of $1 \mathrm{E} 1048.1-5937$ which is the only AXP with a singly-peaked pulse profile and shows no energy dependence (Kaspi et al. 2001; Tiengo et al. 2002).

\section{Pulsed Fractions}

In this section, we quantify the modulations in the pulse profiles using the pulsed fraction defined by

$$
P F=\frac{F_{\max }-F_{\min }}{F_{\max }+F_{\min }},
$$

where $F_{\max }$ and $F_{\min }$ are the maximum and minimum fluxes received by an observer at infinity during a pulse cycle. Bolometric pulsed fractions have been discussed in DeDeo, Psaltis, \& Narayan (2001) for a parametrized form of radially peaked beaming functions, which are not directly applicable to thermally emitting magnetars. Here we present results using the beaming functions calculated for the ultramagnetized neutron stars as well as investigate the dependence of the pulsed fractions on various model parameters. (See also Özel et al. 2001 for an earlier discussion).

Figure 11 shows the dependence of the bolometric pulsed fraction on the relativity parameter $p$ of the neutron star for the case of one and two poles. General relativistic effects alter the observable modulation of X-ray emission originating from a neutron star surface and in general lead to a significant suppression of the pulse amplitude when the gravitational lensing by the star is strong. Therefore, the pulsed fraction is expected to decrease monotonically as the neutron star becomes more relativistic. This is indeed the case seen in the single pole geometry.

The antipodal emission case, on the other hand, shows a more complex behavior. As $p$ decreases, we first obtain the more commonly observed suppression of the pulse amplitudes. A qualitative change occurs at $p \approx 3$ when the lensing by the neutron star is strong enough to lead to the previously discussed enhancement of the flux at the intermediate phases $\phi= \pm \pi / 2$, at which the emission cones intersect (see Fig. 3). This causes the pulsed fraction to increase towards smaller relativity parameters. As $p$ decreases further, nearly the entire surface becomes visible to the observer and thus the behavior is again reversed.

Figure 12 shows the dependence of the pulsed fraction on the size of the emitting region for the two surface temperature distributions. This figure serves as a typical example and summarizes the two important aspects of how the pulsed fraction changes with size: (i) The pulsed fraction falls off much more slowly with increasing angular size of a single pole compared to that of two antipodal hot regions. This results in a much higher pulsed fraction for the same total luminosity (for a fixed effective temperature) in the single pole case since luminosity grows linearly with surface area. As discussed in Özel et al. (2001), this has important implications for the AXPs, which have both high 


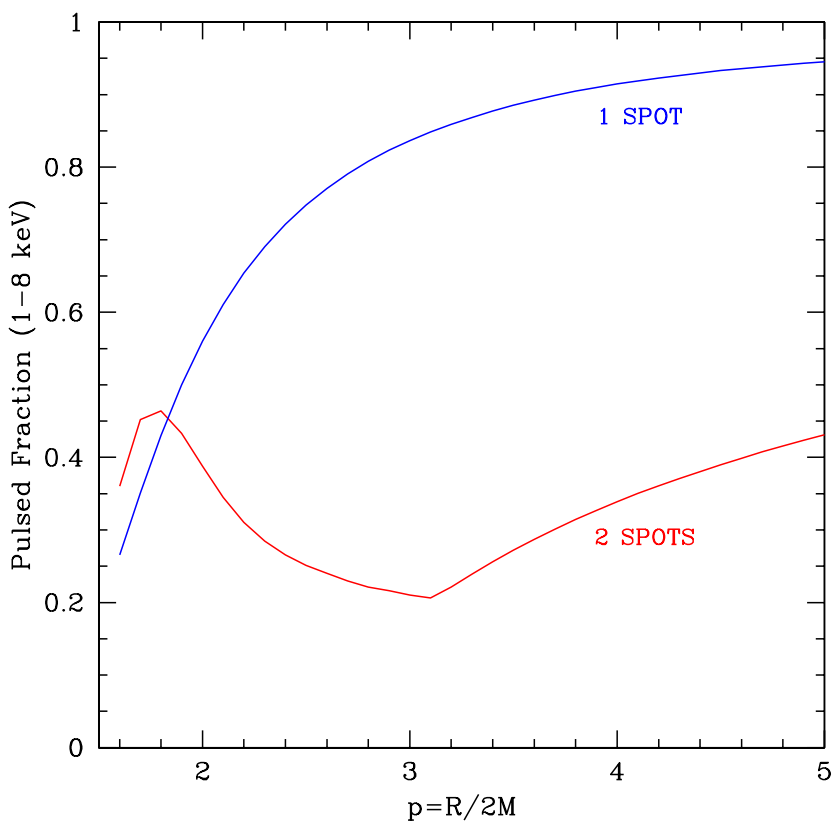

Fig. 11. - The dependence of the $1-8 \mathrm{keV}$ pulsed fraction on the compactness of the neutron star for a single and two antipodal hot regions. In the single pole case, $\alpha=\beta=60^{\circ}$ and $\rho=40^{\circ}$, while in the antipodal emission geometry, $\alpha=\beta=90^{\circ}$ and $\rho=25^{\circ}$.

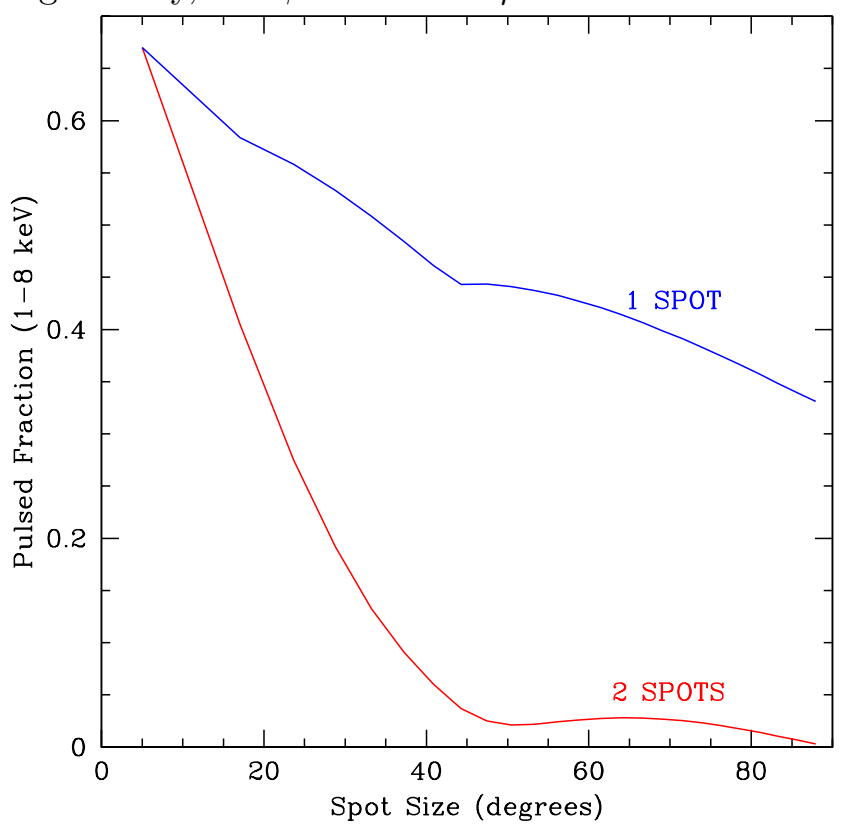

Fig. 12. - The dependence of the pulsed fraction on the size of the emitting region for $p=2.5$ and $\alpha=\beta=50^{\circ}$. Because of gravitational lensing effects, the dependence is non-monotonic. 
pulsed fractions and high inferred luminosities. (ii) The pulsed fraction varies non-monotonically with increasing size of the hot regions. This follows directly from the discussion in $\S 3.2$, where we showed that the pulse profiles go through a sudden change at $\rho \sim 40^{\circ}$ when the non-radial beams from the outer edges of a large hot region are seen simultaneously by an observer, resulting in an enhanced flux. The pulsed fraction, therefore, goes through a corresponding increase when the peak of the pulse profile shifts to $\phi=0^{\circ}$.
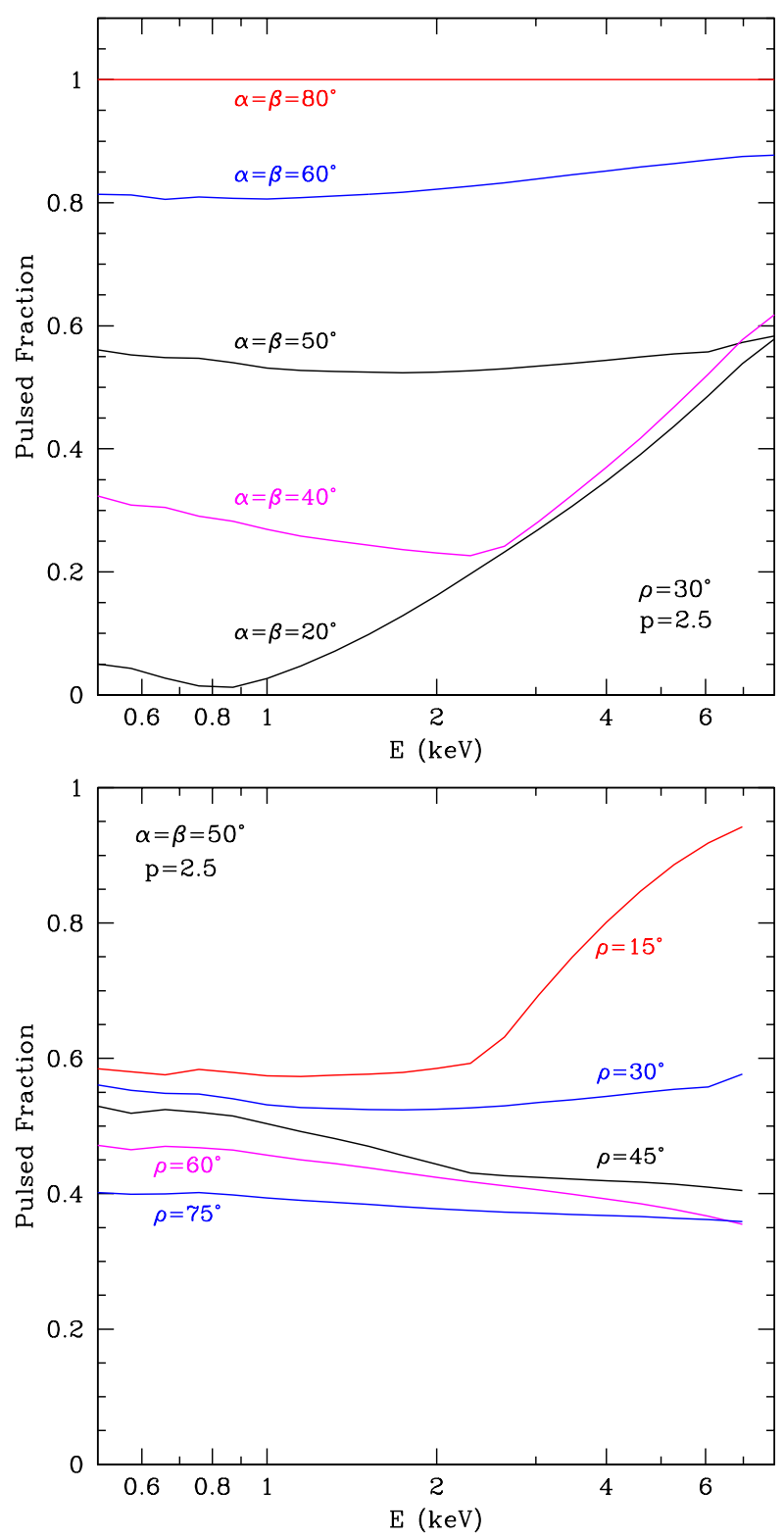

Fig. 13. - The dependence of the pulsed fraction on photon energy for (top panel) a range of observer angles and (bottom panel) a range of sizes of the emitting area when the emission originates from a single hot pole. For most of the parameter space, the dependence is very weak. 
Finally, Figure 13 shows, for the single pole case, the dependence of the pulsed fraction on photon energy for a range of orientation angles $\alpha$ and $\beta$ and for different angular sizes of the hot region $\rho$. For $\rho \gtrsim 20^{\circ}$ and $\alpha$ or $\beta \gtrsim 50^{\circ}$, the pulsed fractions are remarkably constant within the energy range of interest. It is interesting to note that these ranges of parameters cover most of the parameter space of orientation angles for a random distribution and correspond at the same time to the brightest sources because of their large emitting areas. For the other values of the parameters, the pulsed fractions show an increase with photon energy that is also characteristic of the antipodal emission geometry for nearly all parameter values (see Özel et al. 2001 for the corresponding figure in the antipodal emission geometry and a discussion of the physical reasons behind this characteristic shape).

\section{Discussion}

We studied the timing properties of ultramagnetized neutron stars emitting thermally from their surfaces. We used the energy and angle dependence of the emerging radiation obtained from the recent detailed calculations of radiative equilibrium atmospheres in ultrastrong magnetic fields (Özel 2001, 2002). We combined them with the general relativistic calculation of photon transport from the neutron star surface to an observer at infinity and calculated the expected pulse profiles for a wide range of model parameters. We found that the combination of the non-radially peaked beams relevant to magnetars with the strong gravitational lensing leads to a number of qualitatively new and interesting results on the pulse morphology and amplitudes, which we summarize below.

1. An emission geometry consisting of one hot pole gives rise to one or two peaks per pulse cycle, whereas an antipodal geometry can produce one to four peaks, depending strongly on the orientation angles $\alpha$ and $\beta$ as well as on the relativity parameter $p$ and the angular size of the emitting region $\rho$.

2. The pulse profiles in different energy bands show a wide range of morphologies in both emission geometries.

3. The non-radial beaming of the thermal radiation emerging from an ultramagnetized neutron-star surface can give rise to high pulsed fractions even for very compact neutron stars.

4. In the case of an antipodal emission geometry, the pulsed fraction may not be a monotonic function of the relativity parameter $p$, in contrast to the case of radially peaked beaming patterns.

5. The pulsed fraction does not decrease monotonically with the size of the emitting region but shows a secondary maximum at intermediate $\left(\sim 60-70^{\circ}\right)$ angular sizes.

6. The pulsed fraction in the antipodal emission geometry shows a characteristic increase with photon energy in the 1-8 keV range. In contrast, the pulsed fraction in the single pole case has, in general, a very weak energy dependence. 
Note that a number of simplifying assumptions have been made in the models presented here. We have taken the temperature across a hot region to be uniform, which may not be realistic for all the mechanisms that could power the surface emission. In the case of a cooling neutron star with a dipole magnetic field, the temperature is a function of the magnetic latitude and thus shows variations across the surface of the neutron star (e.g., Page 1995). Also in the case of a single hot pole, similar variations can also be expected. This can affect the pulse profiles, possibly reducing the pulsed fractions and smoothing out the pulse morphologies. Similarly, allowing for some cooler emission from the rest of the neutron star can alter the pulse profiles and may account for the additional complexity observed in the energy-dependent pulse profiles of AXPs.

We also assumed that the emission properties are determined entirely by the stellar atmosphere and have not taken into account the possible effects of the neutron star's magnetosphere further above (see, e.g., Thompson, Lyutikov, \& Kulkarni 2002). At present, the structure of the magnetosphere of a strongly magnetized neutron star is not well understood and can only be treated through parametrizations. Note that the alternative class of models of AXPs that rely on accretion onto a magnetized neutron star also require the study of the processes that take place in the accretion column in the neutron star's magnetic field. It was shown earlier that such processes can also lead to a variety of pulse profiles (e.g., Mészáros \& Nagel 1985) but further study is necessary to determine their relevance for AXPs.

The results presented above have direct implications for the thermally emitting magnetar models of AXPs. To carry out a comparison with observations, we summarize the timing properties of these sources which can be used to constrain such models. First, the observed pulse profiles show two prominent peaks per pulse cycle in four sources and a single peak in the fifth source (e.g., Gavriil \& Kaspi 2002). Second, the energy dependence of the pulse morphology can be very weak, as in 1E 1048.1-5937, or quite strong, as in 1RXS 1708-4009 (Gavriil \& Kaspi 2002). Third, AXPs can have pulsed fractions as high as $70 \%$, together with luminosities that are high for their low inferred effective temperatures (Özel et al. 2001). Finally, their pulsed fractions show a weak dependence on photon energy (e.g., Oosterbroek et al. 1998).

All the above properties are hard to account for in a thermally cooling magnetar model (e.g., Heyl \& Hernquist 1998) that has a two-fold symmetry and thus has pulse profiles very similar to those of the antipodal emission geometry. In particular, the number of observed peaks in the pulse profiles as well as the strong energy-dependence predicted in the two-pole models do not reproduce the observations. Furthermore, the pulsed fractions in such a model are in general lower than the values observed in AXPs.

The observations, on the other hand, strongly suggest that AXPs are neutron stars with a single, hot region of ultrastrong magnetic field. A single hot pole can be realized in a number of ways. It may be powered by processes such as crustal cracking, magnetic-field reconfiguration, or decay of magnetic multipoles (e.g., Thompson \& Duncan 1996). It could also arise from an offcentered magnetic dipole that renders one of the poles either not observable or else very close to the 
other pole. All of these possibilities need further theoretical investigations in order to determine the geometry of emission, the total energy output and the lifetime of the temperature asymmetries on the surface. In addition, because the first set of processes are expected to occur randomly, they can produce time-variable pulse profiles. Observations of pulse morphology changes similar to the GINGA observation of $1 \mathrm{E} 2259+586$ (Iwasawa, Koyama, \& Halpern 1992) can help constrain the mechanism powering the thermal emission of AXPs. Finally, investigating the energy dependence of the pulsed fractions in the soft X-rays $(\lesssim 1 \mathrm{keV})$ with Chandra and XMM-Newton as well as in longer wavelengths will extend the baseline over which models can be compared to data and provide the most stringent constraints.

I am grateful to Fotis Gavriil and Vicky Kaspi for sharing with me their AXP data prior to publication and for many stimulating discussions on pulse profiles. I thank Dimitrios Psaltis and Ramesh Narayan for useful suggestions and discussions. I also thank the Institute for Advanced Study, where this work was completed, for their hospitality. This work was supported in part by the NASA Chandra grant GO0-1105B and by the NSF grant AST 9820686. 


\section{REFERENCES}

Alpar, M. A. 2001, ApJ, 554, 1245

Cardall, C. Y., Prakash, M., \& Lattimer, J. M. 2001, ApJ, 554, 322

Chatterjee, P., Hernquist, L., \& Narayan, R. 2000, ApJ, 534, 373

DeDeo, S., Psaltis, D., \& Narayan, R. 2001, ApJ, 559, 346

Gavriil, F. \& Kaspi, V. 2002, ApJ, in press

Heyl, J. \& Hernquist, L. E. 1998, MNRAS, 300, 599

Ho, W. C. G. \& Lai, D. 2001, MNRAS, 327, 1081

Iwasawa, K., Koyama, K., \& Halpern, J. P. 1992, PASJ, 44, 9

Juett, A. M., Marshall, H. L., Chakrabarty, D., Canizares, C. R., Schulz, N. S. 2002, in Neutron Stars in Supernova Remnants, eds P. O. Slane and B. M. Gaensler (ASP Conference Proceedings)

Kaspi, V. M., Gavriil, F. P., Chakrabarty, D., Lackey, J. R., \& Muno, M. P. 2001, ApJ, 558, 253

Mészáros, P. \& Nagel, W. 1985, ApJ, 299, 138.

Oosterbroek, T., Parmar, A. N., Mereghetti, S., \& Israel, G. L. 1998, A\&A, 334, 925

Özel, F. 2001, ApJ, 563, 276

Özel, F. 2002, ApJ, submitted

Özel, F., Psaltis, D., \& Kaspi, V. M. 2001, ApJ, 563, 255

Page, D. 1995, ApJ, 442, 273

Patel, S. K. et al. 2001, ApJ, 563, L45

Tiengo, A., Göhler, E., Staubert, R., \& Mereghetti, S. 2002, A\&A, in press (astro-ph/0111304)

van Paradijs, J., Taam, R. E., \& van den Heuvel, E. P. J. 1995, A\&A, 299, L41

Thompson, C. \& Duncan, R. C. 1996, ApJ, 473, 322

Thompson, C., Lyutikov, M., \& Kulkarni, S. 2002, ApJ, submitted (astro-ph/0110677)

Tsai, W. \& Erber, T. 1975, Phys. Rev. D, 12, 1132

White, N. E., Angelini, L., Ebisawa, K., Tanaka, Y., \& Ghosh, P. 1996, ApJ, 463, L83

Zane, S., Turolla, R., Stella, L., \& Treves, A. 2001, ApJ, 560, 384 